\title{
Eficacia de los aparatos de expansión rápida maxilar Hyrax y Haas
}

\section{Efficacy of the appliances for rapid maxillary expansion Hyrax and Haas}

\section{Eficácia dos aparelhos de expansão rápida da maxila Hyrax e Haas}

\author{
Luis Alberto Vallejo Izquierdo ${ }^{1}$ \\ Jenny Edith Collantes Acuña ${ }^{2}$ \\ Maria Cristina Rockenbach Binz \\ Karla Kamila Vallejo Izquierdo \\ David Eduardo Vallejo Izquierdo ${ }^{3}$ \\ 1 luisvallejo1@hotmail.com \\ 2 jennyc@uhemisferios.edu.ec \\ 3 loshemisferios@gmail.ec \\ Endereço para correspondência: \\ Jenny Edith Collantes Acuña \\ Universidad de los Hemisferios \\ Paseo de la Universidad 300 y Juan Díaz \\ Urbanización Iñaquito Alto \\ Quito - Equador \\ E-mail: jennyc@uhemisferios.edu.ec
}

Recebido: 27.08 .2020

Aceito: 08.09.2020

\section{RESUMEN}

Este estudio evalua la eficacia que tienen los disyuntores dento-muco-soportados Haas y dento-soportados Hyrax, en tratamientos para corregir deficiencias del arco transversal superior, siguiendo los protocolos de activación tuvieron efectos ortopédicos satisfactorios durante la expansión maxilar rápida. Los resultados no tuvieron mayor relevancia entre estos, sin embargo, se recomienda Hyrax por la asepsia para el paciente.

PALABRAS CLAVE: Ortodoncia. Aparatos ortodóncicos. Maxilar.

\footnotetext{
ABSTRACT

This study evaluates the efficacy of the Haas tooth-tissue-borne and Hyrax tooth-borne appliances in treatments to correct transverse deficiencies of the upper arch, following the activation protocols that had satisfactory orthopedic effects during rapid maxillary expansion. The results were not more relevant between them, however, Hyrax is recommended due to the hygiene for the patient.
}

KEYWORDS: Orthodontics. Orthodontic appliances. Maxilla. 


\section{RESUMO}

Este estudo avalia a eficácia dos disjuntores dentomucossuportado Haas e dentossuportado Hyrax, em tratamentos para correção de deficiências transversais do arco superior, seguindo os protocolos de ativação tiveram efeitos ortopédicos satisfatórios durante a expansão rápida da maxila. Os resultados não foram mais relevantes entre estes, porém o Hyrax é recomendado devido à assepsia para o paciente.

PALAVRAS-CHAVE: Ortodontia. Aparelhos ortodônticos. Maxila. 


\section{INTRODUCCIÓN}

La expansión rápida maxilar, es un procedimiento ortopédico mecánico, efectivo en alteraciones maxilares transversales, indicados en mordida cruzada posterior, paladar atrésico, apiñamiento superior y también facilita el flujo del aire nasal al patrón normal'. Los disyuntores fijos liberan fuerzas en el maxilar y los dientes de anclaje, ocasionado la abertura de sutura palatina y cambios en la región alvolear ${ }^{2}$.

Estos aparatos tienen un tornillo de expansión en el centro, ambos implican la adaptación de bandas en los primeros premolares y/o primeros molares. Los disyuntores: Haas, tiene porciones de acrílico colocadas en el paladar convirtiéndolo en muco dento soportado, mientras que; Hyrax es de alambre de metal y permite a cierta distancia del paladar siendo dento soportado, es de fácil elaboración y asepsia para los pacientes ${ }^{3.4}$.

La aplicación de fuertes fuerzas causadas por los tornillos de expansión que oscilan entre 0.9 y $4.5 \mathrm{~kg}$, durante un periodo de 1 a 3 semanas, separan la sutura palatina media ${ }^{5}$.

Algunos estudios indican resultados diferentes o similares en los tratamientos ejecutados a pacientes, sin embargo, no existe un consenso establecido ${ }^{3-4,6}$. Con esta referencia, el objetivo es realizar una revisión de la literatura y comparar los efectos y resultados ortopédicos de la expansión rápida maxilar, con los disyuntores Haas y Hyrax.

\section{REVISIÓN DE LA LITERATURA}

El análisis de los expedientes de varios autores permitirá obtener una idea clara y definida de la mejor opción de los dispositivos de expansión con los debidos protocolos de activación y de acuerdo a la necesidad del paciente.

\section{Historia de los Dispositivos Hyrax y Haas}

La técnica de disyunción maxilar rápida con tornillo de expansión tuvo sus inicios en 1860 con Ángel $^{7}$, siendo cuestionada por dentistas norteamericanos y aceptada por profesionales y académicos europeos como Brown (1914).

Haas investigó la expansión maxilar rápida en animales utilizando procesos estandarizados, los efectos del tratamiento fueron apoyados en análisis histológicos, clínicos, cefalométricos y trasladados en yeso, este método se utiliza hasta en la actualidad ${ }^{5}$.

Según estudios la mordida cruzada posterior, se presenta cuando hay atresia maxilar, la investigación estadística, realizada en niños informa la prevalencia de la mordida cruzada posterior unilateral (11.65\%) la mordida abierta anterior asociada con la mordida cruzada posterior (6.99\%) la mordida cruzada posterior bilateral (1.19\%) y que requieren del tratamiento con expansores ${ }^{8}$.

El procedimiento de expansión maxilar rápida, corrige la atresia maxilar transversal, el efecto principal es la apertura de la sutura intermaxilar por consiguiente el aumento transversal movimientos de la base del hueso superior, sin embargo, con el tratamiento ortopédico una vez que los expansores anclen en los dientes.

Un estudio revela que la expansión rápida maxilar provoca reabsorción de la raíz de los dientes de anclaje bucal y la reabsorción de la placa del hueso bucal a largo plazo?.

El dispositivo Haas irrita la mucosa palatina por empaquetamiento de alimentos, en tal razón presenta el dispotivo Hyrax más higiénico soportado en los dientes, con una configuración dento-soportado ${ }^{10}$.

La sutura palatina media puede osificarse entre 15 y 27 años por consiguiente es adecuado la expansión a los 8 hasta los 15 años (Persson; Thilander, 1977). Sostiene que la osificación se presenta en la edad adulta, estableciendo puentes de osificación en los márgenes óseos iniciando en el sector palatino posterior y evoluciona en sentido antero posterior. En la última década se ha utilizado CBCT en pacientes adultos y jóvenes, diagnosticando que los individuos en pos-puveral presentan diferentes estados de madurez de la sutura palatina media, por tal razón se clasifica dependiendo la etapa de osificación ${ }^{11}$.

Los estudios determinaron que la capacidad regenerativa de la sutura palatina media se da por el estiramiento mecánico causado por el disyuntor ortopédico independiente de la edad, sufriendo una reducción después de la adolescencia. Analizada la imagen radiográfica oclusal se revela que se necesita tres meses para completar la neoformación ósea, sin embargo, a los seis meses se considera confiable debido a la variación individual en el tiempo de osificación de la sutura y un menor efecto de recaída, terminado este periodo se retira el disyuntor.

Una abertura de la sutura palatina media se muestra de forma triangular en sentido anteroposterior con la base hacia la región anterior y en sentido inferior superior con la base hacia la cavidad bucal ${ }^{12}$. Debido a mayor resistencia de las láminas pterigoideas del hueso esfenoide y la rigidez de las suturas óseas zigomáticas, por esta causa se observa un diastema entre los incisivos centrales superiores que se cierra debido a la acción de las fibras transeptales, este proceso aumenta el espacio nasal, facilitando la respiración ${ }^{8,12-17}$.

El efecto ortodóntico del aparato expansor, estima una apertura del $50 \%$ en dentición primaria y mixta y un $65 \%$ en permanente, es decir con el aumento de la edad y la resistencia de la sutura ósea se espera un mayor efecto dental en detrimento de la separación intermaxilar ${ }^{18}$.

Durante la apertura activa de la sutura, los incisivos se separan la mitad de la distancia abierta por el tornillo, se verticalizan e inclinan hacia lingual, debido al estiramiento de los músculos peribucales ${ }^{5}$, al final las fibras transeptales tensan primero las coronas, luego las raíces a sus inclinaciones axiales originales. 


\section{Expansor Haas}

Conformación del aparato - Confeccionado con barras de conexión palatinas con alambre de $1.2 \mathrm{~mm}$ de espesor, soldadas en las dos bandas de cada hemiarco en el primer molar y primer premolar. Los dos botones palatinos de resina acrílica que rodean las barras de conexión de cada cuadrante tocan el paladar, liberando solo las regiones de las papilas gingivales y la rugosidad palatina. El tornillo expansor, que es el elemento activo del dispositivo, se sumerge en la porción acrílica sobre el rafe palatino ${ }^{15}$.

Un detalle mecánico consiste en hacer que el tornillo sea más oclusal, a la altura del tercio cervical de los dientes, de modo que la estructura metálica este menos inclinada y más paralela al plano oclusal.

Anclaje y distribución de fuerzas - El anclaje rígido con soporte dento mucoso, es responsable de la eliminación de los procesos maxilares a medida que se activa el tornillo, genera una fuerza que oscila entre 1000 y 3500 gr en una sola activación y acumula más de 7000 gr durante activaciones consecutivas contra los dientes superiores y el paladar. Este rompe la resistencia ósea y sutural, y separar los procesos maxilares y palatinos a un nivel de sutura palatina mediana ${ }^{8}$. Se considera un periodo de uso de 5 meses para la osificación de la sutura palatina media, la preocupación es el contacto con la mucosa palatina.

Modificación del disyuntor Haas - el aparato utilizado en dentición primaria y mixta contiene solo dos bandas en la región posterior adaptada en el segundo molar permanente. El diente de anclaje anterior, el canino caducifolio, no recibe una banda, sino una extensión de la barra de conexión que abraza este diente similar a una abrazadera "C".

Protocolo de activación - Considera tres cuartos de vuelta, sabiendo que un cuarto de vuelta corresponde a 0.25 $\mathrm{mm}$. De acuerdo con la orientación por grupo de edad, se observa el intervalo de tiempo con la evolución clínica de la expansión con el dispositivo ${ }^{2}$.

- Hasta 14 años de edad: 4 cuartos de vuelta al instalar el aparato, 2 cuartos de vuelta al día, 2 a 4 cuartos de vuelta en el control semanal, duración aproximada de 3 semanas, tornillo expansor abierto de 12 a 16 mm en estabilización, mantener el dispositivo en posición al menos 3 meses.

- De 15 hasta 18 años de edad: 2 cuartos de vuelta al instalar el aparato, 1 cuarto de vuelta por día, 2 semanas entre los controles semanales de 1 a 2 cuartos de vuelta, duración aproximada 6 semanas, abertura del tornillo de 12 a $16 \mathrm{~mm}$ en estabilización, mantener el dispositivo no menos de 3 meses.

- De 18 hasta 25 años: 1 cuarto de vuelta en la instalación, 1 cuarto de vuelta en días alternos, observación cada 4 semanas, duración de 12 a 14 semanas, apertura del tronillo de 12 a 14 mm en estabilización al menos por 3 meses, el ajuste se modifica ocasionalmente dependiendo la comodidad del paciente.

- Después de los 25 años: 1 vuelta en la instalación, y una todos los días, si aumenta la presión suspender la activación, reduciendo a la mitad, es decir, una vuelta cada dos días, si la activación es cada dos días, activar cada tres días o dos veces por semana, el control cada 6-8 semanas, duración aproximada 28-40 semanas, tornillo abierto de 12$14 \mathrm{~mm}$ en estabilización, por lo menos 3 meses.

- Después de los 20 años el protocolo es discutible "cuando mayor es la edad del paciente, peor es el pronóstico" ${ }^{19}$. En el estudio del cierre de las suturas palatinas medias, concluyeron que el cierre se produce a la misma edad, sin embargo, no evoluciona al mismo grado de las demás suturas craneanas, se supone que el final de crecimiento maxilar se produce a los 14 años en mujeres y 17-18 en hombres y comienza la destrucción de la sutura mediana y transversal de la mandíbula.

\section{Expansor HYRAX}

Conformación del aparato - Confecciona el disyuntor con la adaptación de bandas en los primeros molares y premolares superiores permanentes, transferidos a un modelo de yeso que las contiene. El tornillo expansor es posicionado en un pequeño montículo de yeso para mantenerlo alejado del paladar, lateralmente, equidistante de ambos procesos alveolares maxilares y orientado para una activación de anterior a posterior. El marco metálico se caracterizó por la adaptación de segmentos redondos de alambre de acero $0.40 "$ en la superficie vestibular de las bandas y 0.059 " al tornillo expansor y la superficie palatina de las bandas. La soldadura se realizó en las barras vestibulares, palatinas y el tornillo expansor ${ }^{10}$.

Hyrax, es un dispositivo que solo tiene las bandas y el tornillo expansor, estas se unen a los primeros molares y premolares unidos por la superficie vestibular y palatina por medio de alambre de acero inoxidable. El tornillo en expansión revela 4 varillas de alambre de acero fluido fundido, soldada a las cuatro bandas de los molares y premolares.

Hecho con bandas en los primeros molares y primeros premolares, que están unidas por la superficie vestibular y palatina, por medio de un alambre de acero inoxidable y un tornillo expansivo, que tiene cuatro alambres de acero fundido, cada uno de los cuales esta soldado a las cuatro bandas de los molares y premolares.

Anclaje y distribución de las fuerzas - El anclaje de este aparato es puramente dental, la magnitud de las fuerzas, presentan una mayor cantidad de movimiento ortodóntico (movimiento dental) y movimiento ortopédico (movimiento óseo). La ausencia del acrílico palatino, permite una fácil limpieza, siendo aceptado por ortodoncistas y pacientes.

Modificación del disyuntor Hyrax - El nuevo tornillo de expansión prefabricado, presenta barras palatinas gruesas ya soldadas al tornillo, facilitando la confección del dispositivo de expansión higiénico ${ }^{10}$.

Protocolo de activación - Se ejecuta una vuelta del tornillo 
cada día, hasta lograr una sobre corrección de 2 a $3 \mathrm{~mm}$, al final el tornillo se fija, siguiendo los mismos procedimientos realizados con el interruptor automáticos Haas.

El procedimiento de cementación, la elección del ionómero de vidrio es crucial, debido a las propiedades de retener las bandas en la estructura dental y la preventiva de las caries, con la liberación del fluoruro. Antes de la instalar el dispositivo, se debe hacer una profilaxis con cepillo Robinson, una copa de goma, piedra pómez y agua, especialmente en los dientes que sostendrán el Hyrax.

\section{Disyunción en Pacientes con Fisura}

El labio leporino del paladar (ERM), se describe como una malformación congénita que afecta la fisonomía, fonética y maloclusión, estos pacientes sufren una contracción del arco dental superior posterior en el procedimiento quirúrgico por lo que requieren de una expansión maxilar rápida (ideal a los 4 a 6 años). Con este proceso se logra la apertura de la sutura palatina media y mantener su una forma original, un aumento en la cavidad nasal, entre otros.

El dispositivo Hyrax utiliza una fuerza intensa sobre los alveolos del arco superior, no moviliza las piezas dentarias formando un nuevo hueso. Para el control del tratamiento es recomendado rayos $\mathrm{X}$ oclusales, para probar clínicamente la apertura del diastema, que disminuye o aparece en un periodo de 15 a 20 días $^{20}$.

Los efectos de un disyuntor Haas, en 25 pacientes con hendidura unilateral completa, con labio y paladar hendido completo, mordida cruzada posterior y con 27 de mordida cruzada sin labio y paladar hendido, produjo una inclinación de los caninos y dientes posteriores, aumento en las medidas transversales del maxilar, perímetro del arco, volumen y profundidades palatinas, solo disminuyeron las longitudes del arco.

No existieron diferencias relevantes entre los dos grupos, excepto la distancia inter molar una rápida expansión maxilar producen cambios similares en pacientes con hendidura unilateral labio y paladar en comparación con pacientes no agrietados a excepción de la longitud del arco palatal profundidad que disminuyo después de la expansión.

\section{Diferencias de los Expansores}

La principal, es la presencia o ausencia de una parte acrílica cerca del paladar. El aparato Haas tiene una porción acrílica para obtener el máximo anclaje y mayor rigidez, que favorece la distribución de las fuerzas de expansión entre los dientes posteriores y las bases palatinas, mientras que Hyrax, no lo contiene y se fija a los dientes con la ayuda de bandas, llevando la fuerza al maxilar solo a través de los dientes ${ }^{9}$.

Utilizando modelos escaneados y cefalogramas laterales en 19 pacientes, el estudio demostró que el dispositivo Haas presentó una forma de paladar más amplia por la presencia del acrílico y permitió menos inclinación ósea a diferencia del Hyrax que tuvo mayor inclinación alveolar y dental ${ }^{3}$.

Evaluaron 8 pacientes, sometidos a una expansión maxilar ortopédica mediante tomografía computarizada utilizando los dos dispositivos, el resultado es que ambos tenían valores similares, sin embargo, el grupo con el aparato Haas tuvieron valores más altos en relación con la inclinación de los dientes de soporte ${ }^{6}$.

En un estudio de 31 pacientes divididos en dos grupos, con edad promedio de 13 años y dos meses, se solicitó tele radiografías laterales antes del tratamiento, después de la fase de expansión activa y después de completar la nivelación de ortodoncia, se realizaron trazados cefalométricos y análisis de puntos concluyendo que el posicionamiento del maxilar en dirección antero posterior experimento cambios similares para ambos grupos, con la aparición de un avance maxilar en la fase posterior a la disyunción, seguido de un retorno suave después de la nivelación de la ortodoncia. La posición maxilar vertical tuvo cambios similares con la aparición de un desplazamiento vertical del maxilar hacia abajo sin rotación ${ }^{4}$.

En la comparación de los efectos inducidos en la región periodontal efectuada por los disyuntores, concluyeron, que la expansión en sí mismo reduce el hueso en la región vestibular de los dientes de anclaje y aumenta la región palatina. Haas causo una menor expansión de la región del hueso palatino y con Hyrax causo una mayor reducción del hueso bucal en la regios de los premolares de anclaje?

En el estudio de las inclinaciones dentales de 39 pacientes con una edad promedio de 13 años, que se sometieron a una expansión maxilar rápida y divididos en grupos por disyuntor y usando dos tiempos clínicos (antes y una semana después de la fase de la expansión activa) son proyectadas en yeso, donde se traza una línea con sulfato de bario entre las cúspides vestibulares de los primeros molares superiores (derecha izquierda) luego se realizan tomas radiográficas, que visualizaron inclinación molar y el proceso alveolar contiguo.

Los estudios realizados de expansión maxilar mediante tomografías computarizadas de haz cónico, evaluaron que los efectos en anteroposteriores inmediatos por la expansión, existe una luxación maxilar anterior mayor en el grupo de Haas. Y los efectos en transversales inmediatos tiene un comportamiento similar, sin embargo, el dispositivo Hyrax demostró una mejor respuesta ortopedia inicial y con Haas mostro una mayor tendencia a inclinar el anclaje molar hacia vestibular ${ }^{21}$. 


\section{DISCUSIÓN}

En el estudio de los cambios dentoesqueléticos tratados con el dispositivo Haas, el autor enfatizo que está indicado para la corrección de mordidas cruzadas posteriores, con corrección espontánea de las mordidas anteriores, debido al levantamiento de la mordida por el acrílico, debido al corto tiempo en boca ( 3 meses), los resultados no son estadísticamente significativos, pero es justificable al presentar un componente esquelético efectivo ${ }^{22}$. Mediante tomografías computarizadas, demostraron que la sutura palatina se abrió con éxito y las dimensiones transversales tuvieron aumentos significativos después de la expansión maxilar, ambos expansores producen efectos similares ${ }^{6}$.

Realizó el mismo estudio, pero con el disyuntor modificado con soporte dental, concluyó que existió la apertura de la sutura palatina media, sin embargo, luego del periodo de contención (6 meses) la sutura apareció organizada presentando una dimensión similar a la fase de pretratamiento $^{23}$. Tomo datos del estudio anterior concluyó que, el RME produjo aumentos significativos en dimensiones transversales de la mandíbula ${ }^{18}$.

Evaluaron el desplazamiento anteroinferior del maxilar con el uso de Haas, concluyeron que está indicado a casos con patrón de crecimiento desfavorable para la disyunción(dolicofaciales) ya que el dispositivo evita el desplazamiento vertical maxilar, concordando con McNamara y Brundon el aparato causa cambios en la dimensión vertical y modificaciones en la dimensión vertical sagital, indicado para casos dolicofaciales ya que al colocar el acrílico en la parte oclusal de los dientes inhibirá el desarrollo vertical de los dientes posteriores durante el tratamiento ${ }^{24}$.

Compara las alteraciones verticales con el uso de Hyrax y Haas, demostrando que, con el uso del dispositivo unido, había menos movimiento de la columna nasal posterior $\mathrm{y}$ un menor aumento de altura facial anteroinferior en comparación con Hyrax. Con estos datos afirman que Haas es indicado en casos de mayor control vertical ${ }^{25}$.

Los resultados de la alteración dentofaciales verticales obtenidas con los dos expansores, mostraron que el aumento en la distancia inter molar era dos veces mayor que con el dispositivo unido ${ }^{26}$. Las mediciones cefalométricas angulares verticales aumentaron con el expansor de bandas, pero las mediciones lineales para la evaluación de los cambios verticales fueron similares en ambos, ningún dispositivo mostró superioridad en el tratamiento, sin embargo, concluye que existió una expansión ortopédica maxilar no hubo diferencias estadísticas significativas con respecto a los cambios esqueléticos ${ }^{27}$.

Utilizaron disyuntores tipo Haas, Hyrax y pegados encontraron resultados después de la fase activa, que solo el grupo expansor pegado mostró avance desde el maxilar hacia la región anterior siendo menor con los otros dispositivos. También observaron que le dispositivo pegado, utilizado para evitar cambios esqueléticos en la dirección vertical y apertura de la mordida anterior, no estaría justificado, porque al final del periodo de contención, no hubo cambios significativos entre los tres tipos de expansores ${ }^{12}$.

El estudio presentó el aumento de las dimensiones verticales con aparatos expansores colados y en bandas, pero con significado para el grupo con bandas, resultando un mejor control en el grupo unido, con significancia estadística al comparar los grupos ${ }^{28}$.

El tratamiento con los dos dispositivos, declaran que con el expansor Haas produciría expansión con un componente ortopédico mayor (distancia Inter palatal), en cuanto que con Hyrax produciría una expansión con mayor componente dento-alveolar. Esta evaluación se llevó a cabo de tres meses después de la fase activa ${ }^{3}$.

Utilizaron los aparatos Haas y Hyrax en un estudio cefalométrico comparativo sobre la posición vertical y sagital del maxilar después de una expansión maxilar descubrieron resultados similares con el desplazamiento del maxilar en la dirección inferior, sin rotación que se mantiene al final del nivelamiento y un desplazamiento anterior justo después de la disyunción que volvió a los valores previos a la disyunción al final de la nivelación ${ }^{4}$.

Compararon individuos que se sometieron a una expansión maxilar rápida con disyuntores Haas y Hyrax seguidos de un tratamiento estándar de ortodoncia Edgewise, con las que se sometieron solo a un tratamiento de ortodoncia Edgewise y también con personas no tratadas, combinadas por edades y género. Concluye que el tratamiento a largo plazo no influyó en la posición sagital de las bases mandibulares o dimensión vertical. Los cambios cefalométricos desfavorables obtenidos después de la expansión fueron temporales, y, por lo tanto, la consideración sobre el uso de este tratamiento en individuos con patrón de crecimiento vertical con un perfil extremadamente convexo no está justificadas ${ }^{6}$.

Usaron el disyuntor Hyrax y concluyeron que no presentaba, a mediano plazo efectos indeseables, como la inclinación bucal de los dientes posteriores, que debía ocurrir, ya que este dispositivo no tiene soporte mucosoportado como el disyuntos Haas ${ }^{17}$.

Compararon los efectos de la expansión rápida con interruptores automáticos con y sin mucosidad dento, utilizando imágenes computarizadas y concluyeron que ambos expansores mostraron resultados similares. Se observó mayor cambio en la dimensión transversal, fueron insignificantes en la dimensión vertical y anteroposterior, la expansión dentoalveolar fue mayor que la esquelética ${ }^{29}$.

Comparó los efectos del plano transversal, utilizando los dos dispositivos, usando tomografías computarizadas antes de la expansión y al final concluyo que hubo un aumento en la dimensión transversal del maxilar. La expansión esquelética fue menor que la dentoalveolar. 
Hyrax tuvo produjo mayores efectos ortopédicos que el expansor Haas, pero el efecto fue inferior a $0.05 \mathrm{~mm}$ cada lado, no significativo ${ }^{21}$.

La expansión rápida maxilar es un procedimiento ventajoso y estable en el tratamiento de casos de real deficiencia transversal del arco superior y capacidad respiratoria inadecuada. Las dificultades para su ejecución aumentan con la edad debido a la obliteración de la sutura que confiere una resistencia esqueletal significativa, principalmente a nivel de las suturas maxilocigomáticas y maxilofrontales.

El disyuntor Hyrax ha sido identificado como el dispositivo expansor maxilar que mejor permite a los pacientes realizar su higiene. Sin embargo, por no ser dento mucosoportado y no poseer ningún dispositivo de control vertical, se presume que este aparto puede causar en mayor amplitud que los expansores Haas, inclinaciones vestibulares de los dientes de soporte agravando los efectos colaterales en sentido vertical. Por lo tanto, el disyuntos Hyrax debe ser contraindicado para pacientes esqueléticamente divergentes.

El expansor Hyrax, no ofrece ningún dispositivo para el control vertical de los dientes posteriores, como el dispositivo pegado y menor anclaje que el dispositivo Haas.

Durante mucho tiempo, se afirmó que el dispositivo dento mucosoportado tipo Haas promovería un mayor efecto ortopédico y un menor efecto ortodóntico durante la expansión rápida maxilar. El disyuntor dento soportado tipo Hyrax, por otro lado, actuaría directamente sobre la placa del hueso vestibular de los dientes de anclaje porque no posee el anclaje palatal presente en el tipo Haas.

\section{CONCLUSIÓN}

Considerando la revisión de la literatura, se puede concluir que tanto los interruptores dento-muco-soportados como los dento soportados tuvieron efectos ortopédicos satisfactorios durante la expansión maxilar rápida y no fueron encontradas alteraciones significativas entre los dos tipos de disyuntores que demostraran superioridad entre uno de ellos.

\section{REFERENCIAS}

1. Siqueira DF, Almeida RR, Henriques JFC. Avaliação cefalométrica em norma frontal, das alterações dentoesqueléticas produzidas pelo aparelho expansor fixo com cobertura de acrílico. Rev Dental Press Ortodon Ortop Facial. 2002;7(5):27-44.

2. Lima Filho RMA. Alterações na dimensão transversal pela expansão rápida da maxila. R. Dental Press Ortodon Ortop Facial. 2009;14(5):146-57.
3. Oliveira NL, Silveira AC, Kusnoto B, Viana G. Three-dimensional assessment of morphologic changes of the maxilla: a comparison of 2 kinds of palatal expanders. Am J Orthod Dentofacial Orthop. 2004;126(3):354-62.

4. Scanavini MA, Reis SAB, Simões MM, Gonçalves RAR. Avaliação comparativa dos efeitos maxilares da expansão rápida da maxila com os aparelhos de Haas e Hyrax. Rev Dental Press Ortodon Orthop Facial. 2006:11(1):60-71.

5. Haas AJ. The treatment of maxillary deficiency by opening the midpalatal suture. Angle Orthod.1961;35(3):200-17.

6. Garib DG, Henriques JFC, Carvalho PED, Gomes SC. Longitudinal effects of rapid maxillary expansion: a retrospective cephalometric study. Angle Orthod. 2007;77(3):442-8.

7. Ángel EH. Treatment of irregularity of the permanent or adult teeth. Dent. Cosmos. 1860;1:540-44,599-600.

8. Capelozza Filho L, Silva Filho OG. Expansão rápida da maxila: considerações gerais e aplicação clínica. Parte I. Rev Dental Press Ortod Ortop Facial. 1997;2(3):88-102.

9. Garib DG, Henriques JFC, Janson G, Freitas, MR, Coelho AC. Rapid maxillary expansion-tooth tissue-borne expanders: a computed tomography evaluation of dentoskeletal effects. Angle Orthod. 2005;75(4):548-57.

10. Biederman, W. A hygienic appliance for rapid expansion. J Pract Orthod. 1968;2(2):67-70.

11. Angelieri F, Cevidanes LHS, Lorenzo F, Gonçalves JR, Benavides E, McNamara Jr JA. Midpalatal suture maturation: classification method for individual assessment before rapid maxillary expansion. Am J Orthod Dentofacial Orthop. 2013;144(5):759-69.

12. Bramante FS, Almeida RR. Estudo cefalométrico em norma lateral das alterações dentoesqueléticas produzidas por tres expansores: colado, tipo Haas e Hyrax. Rev Dental Press Ortodont Ortop Maxilar. 2002;7(3):19-41.

13. Cavassan AO. Expansão rápida da maxila: avaliação em modelos de gesso. Ortodontia. 1993;26(3):88-92.

14. Cohen M, Silverman E. A new and simple palate splitting device. J Clin Orthod. 1973;7(6):368-9.

15. Souza MM. et al., Análise morfológica do arco superior de portadores de fissura labiopalatal submetidos a diferentes protocolos de expansão rápida maxilar: avaliação das alterações maxilares. Rev Dental Press Ortod Ortop Facial. 2009; 14(5):82-91.

16. Farronato G, Giannini L, Galbiati G, Maspero C. Comparison of thr dental and skeletal effects of two different rapid palatal expansion appliances for correction of maxillary asymmetric transverse discrepancies. Minerva Stomatol. 2012;61(3):45-56.

17. Ferreira CMP, Ursi W, Atta JY, Lyra MCO, Lyra FA. Efeitos dentais e esqueletais mediatos da ERM utilizando o disjuntor Hyrax. Rev Dent Press Ortodont Ortop Facial. 2007;12(4):36-48.

18. Ballanti F, Lione R, Fanucci E, Franchi L, Baccetti T, Cozza P. Immediate and postretention effects of rapid maxillary expansion investigated by computed tomography in growing patients. Angle Orthod. 2009;79(1):24-9.

19. Haas AJ. Palatal expansion: just the beginning of dentofacial orthopedics. Am J Orthod. 1970;57(3):219-55. 
20. Machado R, Bastidas M, Arias E, Quirós O. Disyunción maxilar con la utilización del expansor tipo Hyrax en pacientes con labio y paladar hendidos. revisión de la literatura. Rev Latinoam Ortod Odontop. 2012:428-30.

21. Weissheimer A, Menezes LM, Mezomo M, Dias DM, Lima SEM, Rizzarro SMD. Immediate effects of rapid maxillary expansión with Haas-type and hyrax-type expanders: a randomized clinical trial. Am J Orthod Dentofacial Orthop. 2011;140(3):366-76.

22. Spolyar JL. The desing, fabrication, and use of a full-covered bonded rapid maxillary expansión appliance. Am J Orthod Dentofac Orthoped. 1984;86(2):136-45.

23. Lione R, Ballanti F, Franchi L, Baccetti T, Cozza P. Treatment and posttreatment skeletal effects of rapid maxillary expansion studied with low-dose computed tomography in growing subjects. Am J Orthod Dentofacial Orthop. 2008;134(3):389-92.

24. Saver DM Johnston MW. Skeletal changes in vertical and anterior displacement of the maxila with bonded rapid palatal expansion appliances. Am J Orthod Dentofacial Orthop. 1989;95(6):462-6.

25. Asanza S, Cisneiros GJ, Nieberg LG. Comparison of hyrax and bonded expansion appliances. Angle Orthod. 1997;67(1):15-22.

26. Reed N, Ghosh J, Nanda RS. Comparison of treatment outcmes with banded and RPE appliances. Am J Orthod Dentofacial Orthop. 1999;116(1):31-40.

27. Mazzieiro ET. Estudo cefalométrico, em norma frontal, das alterações dentoesqueléticas após a expansão rápida da maxila, em pacientes na faixa etária de 10 a 16 anos e 2 meses [dissertation]. Bauru (SP): Universidade de São Paulo; 1994.

28. Galon GM, Calçada F, Ursi W, Queiroz GV, Atta JY, Almeida GA. Comparação cefalométrica entre os aparelhos de ERM bandado e colado com recobrimento oclusal. Rev Dent Press Orthodon Ortop Facial. 2003;8(3):49-59.

29. Lagravére MO, Carey J, Heo G, Toogood RW, Major PW. Transverse, vertical and anteroposterior changes from boneanchored maxillary expansion vs traditional rapid maxillary expansion: a randomized clinical trial. Am J Orthod Dentofacial Orthop. 2010; 137(3):304.e1-12; discussion: 304-5. 